\title{
METAL-INSULATOR TRANSITION IN DOPED SEMICONDUCTORS
}

\author{
J. JAROSZYŃSKI \\ Institute of Physics, Polish Academy of Sciences \\ Al. Lotników 32/46, 00-668 Warszawa, Poland
}

\begin{abstract}
A survey is given of different kinds of metal-insulator transitions (MIT) in doped semiconductors. The role of electron-electron Coulomb interactions and of disorder is discussed vis-a-vis millikelvin experimental results for semimagnetic semiconductors (SMSC) in the vicinity of MIT. Critical behavior of conductivity tensor components and dielectric susceptibility at the magnetic field-induced MIT in p-type $\mathrm{Hg}_{1-x} \mathrm{Mn}_{x}$ Te is compatible with the model in which the MIT is a result of quantum localization driven by disorder-modified electron-electron interactions. At the same time the critical behavior of the Hall coefficient suggests that, in addition to electrons forming the Fermi liquid (FL) and undergoing localization at the MIT, there is certain a concentration of local electron moments, even on the metallic side of the MIT. The formation of these moments can presumably be described in terms of a disordered Hubbard-Mott model.
\end{abstract}

PACS numbers: $71.30 .+h$

\section{Models of the metal-insulator transitions}

The nature of the metal-to-insulator transition (MIT) has attracted much interest for many years. Ever since quantum mechanics was applied to the difference between a metal and an insulator by Wilson (1931) [1], it has been apparent that MIT could occur if any parameter such as volume or composition was changed. For instance, in a crystalline insulator (i.e., according to Wilson, a solid with all bands filled or empty) in which the gap is indirect, decreasing the volume could make the gap shrink to zero and change sign - so the material becomes a metal (a solid with partially filled bands). A transition of this kind, the so-called Bloch transition, is observed in ytterbium under pressure [2].

Here we focus on MIT in doped semiconductors $[3,4]$. In such systems there is a critical impurity concentration $n_{c}$, below which the zero-temperature conductivity vanishes $\sigma(0)=0$ and the material becomes an insulator. Above $n_{c)} \sigma(0) \neq 0$ 
- the semiconductor behaves like a metal. As it is demonstrated experimentally, $n_{c}$ depends mainly on the effective Bohr radius $a_{\mathrm{B}}: n_{\mathrm{c}}^{1 / 3} a_{\mathrm{B}} \approx 0.25$, but it could be changed by external perturbation, such as pressure, magnetic field etc.

\subsection{Hubbard-Mott transition}

Historically, the fact that material with the highest band half-filled (e.g., with one electron per atom) could become an insulator, was anomalous from one-electron band theoretical point of view. Even the name "metal-to-insulator" transition (instead of insulator-to-metal) expresses this anomaly. Here the key role was played by the Mott's paper [5]. He took into account Coulomb electron-electron correlation energy into account. Electrons correlate their motions in order to avoid configurations with two electrons residing on the same site. This is due to an increase of potential energy $E_{\mathrm{p}}$ by Hubbard intrasite energy $U$ in this situation. The amount by which band theory overestimates the ground-state energy of the system due to the neglect of electron correlations is called the correlation energy. The point embodied by the Mott transition is that the correlation energy can, under certain conditions, cause a solid to have an insulating ground state when the neglect-of-correlation band picture would erroneously predict it to be a metal.

In terms of the wave functions of electrons at the Fermi level, the MIT marks a transformation from extended states into localized states. Localization increases the wave-function oscillations and thus increases the kinetic-energy $E_{\mathrm{k}}$ contribution to the total energy. Therefore, quite generally, the MIT will occur provided that the localization results in such a drop of the potential energy which overcompensates the above mentioned increase in the kinetic energy. The Mott transition is an exemplary embodiment of a recurrent theme in solid-state physics: electron localization in the low-density limit signals the triumph of $E_{\mathrm{p}}$ over $E_{\mathrm{k}}$ in that regime.

\subsection{Wigner crystallization}

An earlier version of this theme is the idea of the Wigner lattice. Wigner proposed (1934) [6] that a low-density low-temperature electron gas would crystallize, with the electrons becoming localized and forming a lattice. In this model the MIT is also driven by the Coulomb interactions among electrons, but the impurities are assumed to act only as a uniformly charged neutralizing background. Both Mott and Wigner models deal with crystalline systems.

\subsection{Percolation transition}

Another two models deal with disordered systems i.e. systems without long-range order. The first of them is the percolation model of MIT. Imagine classical particles placed in a randomly fluctuating potential produced by impurities. If the amplitude of the potential fluctuations increases, the particles tend to localize in the "valleys" of the potential, and the volume occupied by them shrinks. If this 
volume turns to be less than $\sim 16 \%$ [7] of the sample volume, the material becomes an insulator. The percolation model disregards the effect of electron-electron interactions as well as quantum phenomena, such as tunneling and interference, which tend to delocalize and localize electrons respectively. The percolation picture can be viewed as a classical limit of the Anderson model of MIT.

\subsection{Anderson transition}

Anderson treats electrons quantum-mechanically. In his famous paper from 1958 [8] he showed that if disorder is strong enough, all of the states in the band are localized. The model is a quantum-mechanical calculation that invokes a tight-binding independent-electron picture. The independent-electron aspect is important and poses the question: "Which mechanism, electron-electron interactions or disorder, plays the dominant role in MIT?". Triumph of the Landau theory [9] of the Fermi liquid (FL) which explained transport properties of the electron in metals suggested that e-e interactions were of minor importance.

Our understanding of the MIT in disordered systems has progressed considerably over the past decade. In particular, it became evident that MIT in disordered systems should be regarded as the quantum localization of the FL induced by scattering. It occurs due to the collective action of many scattering centers and would take place even if the potential of a single impurity, or defect, had no bound states.

It appears, by now, that there coexist two distinct mechanisms by which scattering may give rise to the localization $[4,10,11]$. The first of them, the quantum interference of scattered waves, can be ascribed to single particle diffusive motion. It is known that, when the positions of scattering centers are spatially correlated (as, e.g., atoms in a crystal), the interference enhances or diminishes the transition probability between two points in space depending on the electron wavelength. If, on the other hand, the scattering centers are distributed randomly, the interference terms will vanish after averaging all possible configurations of scattering centers. In other words, the mean transition probability between two points is well approximated by the sum of probabilities of all possible electron paths, in agreement with the classical Boltzmann description of diffusion. However, this is no longer true if we consider the probability of returning to the starting point. This is because the "optical paths", and hence the phases of the transition probability amplitudes, are identical for the clockwise and counterclockwise trajectories of an electron wave along the same sequence of scattering centers. Since such interference is constructive, it increases the probability of return and thus, diminishes the conductivity.

Another consequence of scattering by impurities is the substantial modification of the interactions between electrons. A simplified picture behind it is that the diffusion motion allows two quasiparticles to meet and interact several times. If the two quasiparticles have similar energies, they will acquire a small phase difference between the successive meetings. In such a case the successive interaction events cannot be regarded as independent. This invalidates several conclusions of 
the standard FL theory and, in particular, leads to corrections to the Boltzmann conductivity.

The role played by the above quantum effects grows with the increase of $\hbar / \varepsilon_{\mathrm{F}} \tau$, where $\tau$ is the momentum relaxation time. Since, according to the BrooksHerring formula (and its modification by Moore [12]) $\varepsilon_{\mathrm{F}} \tau$ for ionized impurity scattering depends only on $n^{1 / 3} a_{\mathrm{B}}$, the model in question is compatible with the proportionality of $n_{c}$ to $a_{\mathrm{B}}^{-3}$. An interesting aspect of these quantum corrections is their unusual sensitivity to temperature and to the so-called symmetry lowering perturbations. The corrections depend on the inelastic scattering processes (appearing at $T>0, \tau_{\mathrm{in}} \sim T^{-p}$, where $p \geq 1$ ) as well as on the magnetic field, spin-orbit- and spin-disorder scattering, because these change the phase of the wave functions. The contribution from the electron-electron interactions is affected additionally by thermal broadening of the Fermi-Dirac distribution function, and by the spin-splitting, as the latter influences the time-evolution of the phase difference if the two quasiparticles come from different spin subbands. Depending on the symmetry lowering perturbations present (magnetic field, spin-splitting, spin-orbit- or spin-disorder scattering) various universality classes have been identified [11], each characterized by a different set of critical exponents controlling the behavior of relevant variables in the immediate vicinity of the MIT.

\section{Critical region and exponents}

In this paper we shall show how studies of the critical region can help to determine the dominant driving mechanism of the MIT in doped semiconductors. When physical quantities have a discontinuity at the phase transition, one says, following Landau, that the transition is of the first order [13]. Hubbard-Mott and Wigner transitions are presumably of this kind. When there is no such discontinuity, the transition is of the second order. In the last case, in a region very close to the critical point, i.e. $\left|y-y_{\mathrm{c}}\right| / y_{\mathrm{c}} \ll 1$, one observes quantities obeying power laws:

$$
z \propto\left(y-y_{\mathrm{c}}\right)^{\alpha} .
$$

Here $y$ is a parameter that controls transition and $y_{\mathrm{c}}$ - the value at the critical point. The exponent $\alpha$ and others like that defined in the vicinity of a given critical point, are called critical exponents. Such behavior implies that physical quantities depend nonanalytically on their variables, i.e. on the temperature, the field etc. In such circumstances the usual perturbation theory methods prove ineffective. What is remarkable is that such behavior, though singular, has some very simple characteristic. Different systems, undergoing the most varied kinds of transition can be divided on the basis of their Hamiltonian symmetry consideration into a small number of universality classes, each specified by a certain set of values of the critical exponents. Note, in particular, that within a given universality class the values of critical exponents do not depend on the type of parameter that induces the transition. Moreover, one observes between the critical exponents some very simple relations, called scaling laws, whose degree of universality is even greater. The most powerful tool in physics of phase transitions is the method of 
renormalization group. It allows to reduce the number of degrees of freedom with the aid of a family of continuous transformations in space - increasing the length scale $L$ which eliminates short-wave fluctuations since the inverse of $L$ acts as cutoff parameter for large momenta.

\section{Experimental results and discussion}

In this paper we discuss the application of semimagnetic semiconductors (SMSC) [14] for studying the physics of the MIT. Very accurate information on the critical region can be obtained if the MIT is tuned by an external perturbation. As it is shown in Fig. 1, p-HgMnTe undergoes an insulator-to-metal transition when the magnetic field gets stronger.

Parameters obtained from fits of the extrapolated

TABLE

$T=0$ experimental data by the formula $X=$ $X_{\mathrm{c}}\left|H / H_{\mathrm{c}}-1\right|^{\delta}$ in $p-\mathrm{Hg}_{0.92} \mathrm{Mn}_{0.08} \mathrm{Te}$.

\begin{tabular}{cc|c|c|c}
\hline \hline & $X$ & $X_{\mathrm{c}}$ & $H_{\mathrm{c}}(\mathrm{kOe})$ & Critical exponent $\delta$ \\
\hline$\sigma_{x x}$ & $\left(\Omega^{-1} \mathrm{~cm}^{-1}\right)$ & $9.5 \pm 0.5$ & $15.1 \pm 0.2$ & $1.05 \pm 0.1$ \\
$\sigma_{x y}$ & $\left(\Omega^{-1} \mathrm{~cm}^{-1}\right)$ & $31.2 \pm 5$ & $15.1 \pm 0.2$ & $1.89 \pm 0.1$ \\
$\sigma_{z z}$ & $\left(\Omega^{-1} \mathrm{~cm}^{-1}\right)$ & $1.6 \pm 0.2$ & $14.9 \pm 0.2$ & $1.06 \pm 0.1$ \\
$R_{\mathrm{H}}$ & $\left(\mathrm{cm}^{3} \mathrm{C}^{-1}\right)$ & $790 \pm 70$ & $13.1 \pm 0.2$ & $-0.9 \pm 0.1$ \\
$4 \pi \chi$ & & $60^{a}$ & $15.0 \pm 0.3$ & $-1 . \pm 0.2$
\end{tabular}

${ }^{a}$ the error in the absolute value of $\chi_{\mathrm{c}}$ is probably of the order of $100 \%$.

It can be seen that the transition is continuous. This contradicts the theoretical predictions for both Hubbard-Mott and Wigner transitions, at least in 3d ordered systems. At the same time the continuous MIT is expected if it is driven by disorder. In the metallic phase, i.e. for $H>H_{c}$, where $H_{c}$ is a critical field, the conductivity can be described by scaling formula $\sigma \propto\left(H-H_{\mathrm{c}}\right)^{\nu}$ with the critical exponent $\nu \approx 1$. This value is in apparent disagreement with $\nu=1.8 \pm 0.2 \mathrm{ex}-$ pected from the percolation theory [15], as well as with $\nu=0.5$ [16] derived from one-electron theory of Anderson transition in the magnetic field. On the other hand $\nu=1$ is in accordance with these theoretical predictions for Anderson localization which take into account the effect of scattering-modified electron-electron interactions [11], provided that spin effects which lower the Hamiltonian symmetry exist.

Because of giant spin-splitting characteristic of SMSC semiconductors, the majority of carriers reside in one spin subband. Renormalization group equations for the transition occurring in a totally spin-polarized electron gas (so-called "spin-polarized" universality class in which strong spin-splitting determines the symmetry of system Hamiltonian), taking into account disorder modified e-e interactions [11] indeed predict $\nu=1$, as found experimentally. Moreover, they give a temperature dependence of $\sigma$ in the form $\sigma(T)=\sigma(0)+A T^{\alpha}$ with $\alpha \approx 0.5$, 


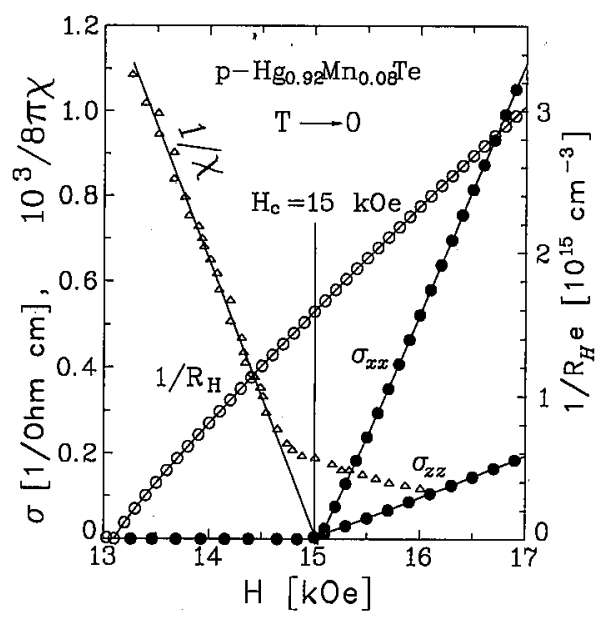

Fig. 1. Extrapolated to $T=0$ values of conductivity tensor components $\sigma_{x x}$ and $\sigma_{z z}$ as well as the inverse of the Hall coefficient $R_{\mathrm{H}}$ and of the dielectric susceptibility $\chi$ measured near the insulator-to-metal transition. Solid lines represent critical behavior with parameters displayed in Table.

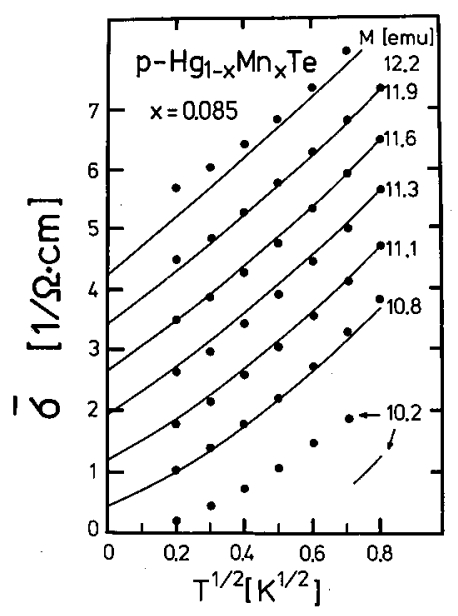

Fig. 2. Conductivity of $p-\mathrm{Hg}_{0.815} \mathrm{Mn}_{0.085} \mathrm{Te}$ vs. square root of temperature in various magnetic fields [17]: 
which is observed on the metallic side of the transition [17], as it is shown in Fig. 2.

In addition to the resistance measurements - the dielectric susceptibility $\chi$ (dielectric constant $\varepsilon=\varepsilon_{\mathrm{s}}+4 \pi \chi$, where $\varepsilon_{\mathrm{s}}$ is the lattice dielectric constant of the host material) and the Hall coefficient $R_{\mathrm{H}}$ have been examined near the MIT in SMSC.

The ability of a metal to screen the Coulomb interaction totally at large distances is an important characteristic of the conducting state of matter. This is related to the wave-vector $q$-dependent static dielectric constant $\varepsilon(q, \omega=0)$ diverging as $q^{-2}$ when $q \rightarrow 0$. On the insulating phase, however, $\lim _{q \rightarrow 0} \equiv \varepsilon_{0}$ is finite. Near continuous MIT, one would thus expect a diverging $\varepsilon_{0} \propto\left(y_{c}-y\right)^{-\zeta}$ from the insulating side. The one-electron Anderson's model of MIT predicts that $\varepsilon_{0}$ should diverge with twice the exponent characterizing the vanishing of the conductivity from the metallic side, i.e. $\zeta=2 \nu[18]$. On the other hand the theory, with e-e interactions included, expects for spin-polarized universality class a universal relation $[11,19]$ between $\zeta$ and the exponent $\alpha$ describing the temperature dependence of the conductivity at $y=y_{\mathrm{c}}: \zeta=1 / \alpha-1$.

To determine dielectric susceptibility the complex conductance $Y=G+\mathrm{i} \omega C$ was measured by using a three probe a.c. bridge. The measurements were performed on the sample used previously for conductivity investigation. After etching, thin indium layers were evaporated on opposite faces of one of the samples, forming a capacitor with area $\sim 5 \mathrm{~mm}^{2}$ and thickness $\sim 300 \mu \mathrm{m}$.
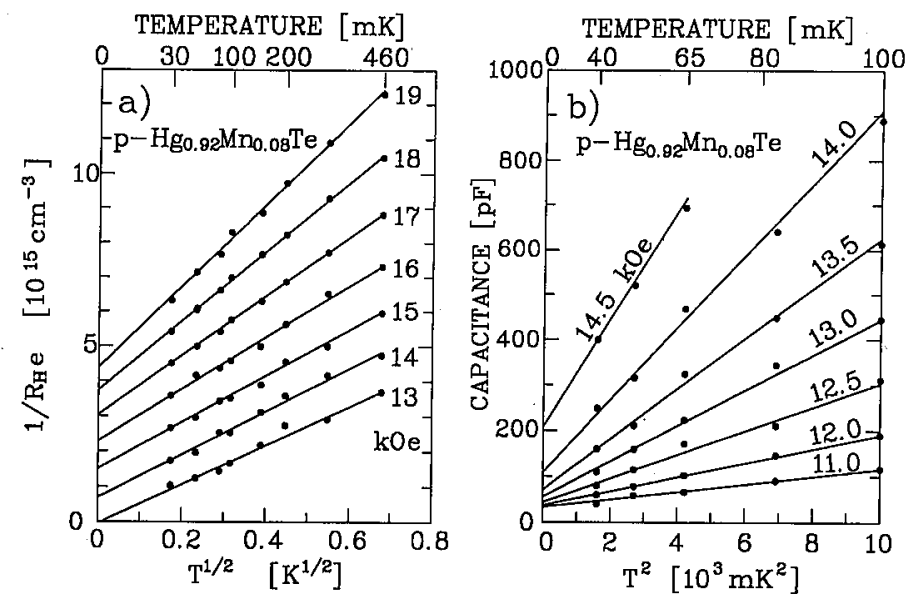

Fig. 3. (a) Inverse Hall coefficient vs. square root of temperature and (b) the parallel equivalent circuit capacitance vs. square of temperature in various magnetic fields. The latter gives the capacitance of the sample in series with that of Schottky contact barrier. Straight-line fits indicate $T^{1 / 2}$ and $T^{2}$ dependences for $1 / R_{\mathrm{H}}$ and $C$, respectively. Results at the lowest measured temperature give values of $R_{\mathrm{H}}$ and $\chi$ close to those found by extrapolation to $T=0$. 
Figure 3(a) presents the parallel equivalent circuit capacitance vs. square of temperature in various magnetic fields. It is seen that the dielectric susceptibility has a strong $T^{2}$ dependence below $100 \mathrm{mK}$. This is in qualitative agreement with the theoretical predictions which take into account the contribution from phonon-assisted processes [20]. It is seen in Fig. 1 that $\chi$ strongly increases when approaching the MIT from the insulating side. However, it does not diverge to infinity, most probably, because of the presence of the Schottky barrier capacitance $C_{\mathrm{S}} \approx 1 \mathrm{nF}$. If the region where $C_{\mathrm{s}}$ becomes important is disregarded, the critical field deduced from the $\chi$ on $H$ dependence coincides with that given by $\sigma(H)$ measurements, $H_{c} \simeq 15 \mathrm{kOe}$. Furthermore, the dependence of $1 / \chi(H)$ is seen to be essentially linear on the magnetic field. This implies $\zeta=1 \pm 0.2$, which gives $\alpha=0.5 \pm 0.1$. The latter is to be compared to the value deduced from the $\sigma$ vs. $T$ dependence, $\alpha=0.65_{-0.2}^{+0.2}[17]$. It should be noted, however, that the experimental data are perturbed by a Schottky barrier capacitance whose field dependence is unknown.

The question: "Does the Hall coefficient exhibit critical behavior approaching the MIT?" has attracted many theoretical and experimental efforts recently. Both non-interacting [21] and interacting [22] models of MIT predict a scaling behavior of $\sigma_{x y}: \sigma_{x y} \propto\left(y-y_{\mathrm{c}}\right)^{\nu_{\mathrm{H}}}$ where $\nu_{\mathrm{H}}=2 \nu$. Since $R_{\mathrm{H}} \propto \sigma_{x y} / \sigma_{x x}^{2}$ the Hall coefficient $R_{\mathrm{H}}$ should not vary critically when approaching the MIT from the metallic side. There are indeed measurements [23] of $R_{\mathrm{H}}$ which are in agreement with these predictions. However, other data [24] give evidence for the critical behavior of $R_{\mathrm{H}}$. In an effort to resolve this issue we have performed the Hall-coefficient measurements in SMSC.

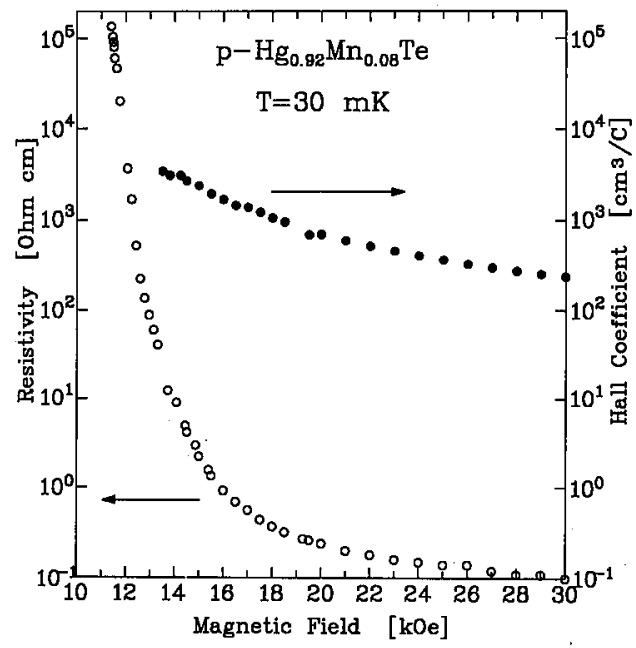

Fig. 4. Resistivity and Hall coefficient in $T=30 \mathrm{mK}$ vs. magnetic field in $p-\mathrm{Hg}_{0.92} \mathrm{Mn}_{0.08} \mathrm{Te}$ in the vicinity of MIT which takes place in $H=15 \mathrm{kOe}$ in this sample.

It is seen in Fig. 4 that resistivity of $p-\mathrm{HgMnTe}$ in $T=30 \mathrm{mK}$ increases much 
stronger than $R_{\mathrm{H}}$ when approaching the MIT from the metallic side. It indicates that $R_{\mathrm{H}}$ varies smoothly in the critical field $H_{\mathrm{c}}\left(\sigma_{x x}\right)$, where conductivity vanishes critically. Since MIT occurs in classically weak fields $\sigma_{x x}>\sigma_{x y}$, the latter implies that the critical exponent of $\sigma_{x y}$ to be twice as that for $\sigma_{x x}$. On the other hand, as it is seen in Figs. 1 and 3(b) the Hall coefficient does exhibit critical behavior. However, the critical field $H_{\mathrm{c}}\left(R_{\mathrm{H}}\right)$ turns out to be substantially lower than critical fields for $\chi(H)$ and $\sigma(H), H_{\mathrm{c}}\left(R_{\mathrm{H}}\right) \approx 13 \mathrm{kOe}$. This behavior of $R_{\mathrm{H}}$ contradicts the simple freeze-out picture in the framework of which the carrier concentration should vanish at the same field as the conductivity.

It has been suggested $[25,26]$ that the field at which $\sigma_{x x}, \sigma_{x y}, \sigma_{z z}$ and $1 / \chi$ tend to zero marks the quantum localization of the FL. According to this model, FL-type electronic states remain valid in the insulating phase, at least at small length scales, where metallic and insulating phases are undistinguishable. On the other hand, it is well-known that deep in the insulating phase the electrons are bound to individual impurities forming localized magnetic moments, due to the Hubbard energy $U$. Obviously, in the last case the electronic states cannot be described within the FL formalism at any length scale. Moreover, there is a growing evidence that such local moments coexist with FL-type states already on the metallic side of the MIT [27]. In the framework of this two-fluid model, it has been speculated [25] that the divergence of the Hall coefficient, visible in Figs. 3 and 4, marks the point where the FL electrons totally disappear. The influence of local moments in SMSC is strongly enhanced by the presence of magnetic ions, which can be polarized via $s$ - $d$ interaction. This, in turn, increases the binding energy and speeds up transformation of the Fermi-states into localized moments. This may explain why the critical behavior of $R_{\mathrm{H}}$ has not been observed in other MI systems.

Most of experimental results are consistent with the picture of the MIT in doped semiconductors as quantum localization of the Fermi liquid by scattering modified e-e interactions. At the same time critical behavior of the Hall coefficient appears to be incompatible with the present theory, and suggests that elements of Hubbard-Mott model are of relevance.

\section{Acknowledgment}

The author is grateful to Prof. T. Dietl, without whom the present work would not be possible, as well as to A. Lenard, W. Plesiewicz, M. Sawicki, T. Wojtowicz and J. Wróbel for fruitful collaboration.

\section{References}

[1] A.H. Wilson, Proc. R. Soc. A 133, 458; 134, 277 (1931).

[2] D.B. McWhan, T.M. Rice, P.H. Schmidt, Phys. Rev. 177, 1063 (1969).

[3] for review see, e.g., The Metal-Non-Metal Transition in Disordered Systems, Eds. L.R. Friedman, D.P. Tunstall, SUSSP Publ., Edinburgh 1978.

[4] see, e.g., Anderson Localization, Eds. T. Ando, H. Fukuyama, Springer, Berlin 1988. 
[5] N.F. Mott, Proc. Phys. Soc. A 62, 416 (1949).

[6] E. Wigner, Phys. Rev. 46, 1002 (1934).

[7] R. Zallen, The Physics of Amorphous Solids, Wiley, New York 1983.

[8] P.W. Anderson, Phys. Rev. 109, 1492 (1958).

[9] L.D. Landau, Zh. Eksp. Teor. Fiz 30, 1058 (1956); 32, 59 (1957); 34, 262 (1958); for reviev see D. Pines, P. Nozieres, Theory of Quantum Liquids, Benjamin, Menlo Park 1966.

[10] for review see, e.g., B.L. Al'tshuler, A.G. Aronov, D.E. Khmelnitskii, A.I. Larkin, in Quantum Theory of Solids, (Ed. I.M. Lifshits), Mir Publishers, Moscow 1982, p. 130; P.A. Lee, T.V. Ramakrishnan, Rev. Mod. Phys. 57, 287 (1985); B.L. Al'tshuler, A.G. Aronov, in: Electron-Electron Interactions in Disordered Systems, Eds. A.L. Efros, M. Pollak, North-Holland, Amsterdam 1985, p. 1; H. Fukuyama, ibid. p. 155.

[11] A.M. Finkel'stein, Zh. Eksp. Teor. Fiz. 86, 367 (1984); C. Castellani, C. Di Castro, P.A. Lee, M. Ma, Phys. Rev. B 30, 527 (1984); C. Di Castro, in [4], p. 96; G. Kotliar, in [4], p. 107; T. Dietl, M. Sawicki, J. Jaroszyński, T. Wojtowicz, W. Plesiewicz, A. Lenard, in: Proc. 19th Internat Conf. on Physics of Semicond., Warsaw 1988, Ed. W. Zawadzki, Institute of Physics, Warsaw 1988, p. 1189.

[12] E.J. Moore, Phys. Rev. 160, 607 and 618 (1967).

[13] for review of phase transition theory, renormalization group method etc. see, e.g. P. Pfeuty, G. Toulouse, Introduction to the Renormalization Group and to Critical Phenomena, Wiley, New York 1977; S. Ma, Rev. Mod. Phys. 45, 589 (1973).

[14] for general properties of Mn-based semimagnetics see, e.g. Diluted Magnetic Semiconductors, (volume Eds. J.K. Furdyna, J. Kossut) in Semiconductors and Semimetals, Vol. 25 (series Eds. R.K. Willardson, A.C. Beer), Academic Press, Boston 1988; for review of transport properties see J. Kossut, ibid. p. 183; N.B. Brandt, V.V. Moshchalkov, Adv. Phys. 33, 193 (1984); Y. Shapira, in: Diluted Magnetic (Semimagnetic) Semiconductors, Symp. Proc. of Mat. Res. Soc., Vol. 97, Eds. S. von Molnar et al., MRS, Boston 1987 p. 209; T. Dietl, M. Sawicki, T. Wojtowicz, J. Jaroszyniski, W. Plesiewicz, L. Świerkowski, J. Kossut, in [4], p. 58; T. Dietl, J. Crystal Growth 101, 808 (1990).

[15] see, e.g., Y. Song, T.W. Noh, S.--I. Lee, J.R. Gaines, Phys. Rev. B 33, 904 (1986).

[16] K.B. Efetov, Adv. Phys. 32, 53 (1983), and references therein; A. Kawabata, Solid-State Electron. 28, 131 (1985).

[17] T. Wojtowicz, T. Dietl, M. Sawicki, W. Plesiewicz, J. Jaroszyński, Phys. Rev. Lett. 56, 2419 (1986).

[18] T.F. Rosenbaum, K. Andres, G.A. Thomas, R.N. Bhatt, Phys. Rev. Lett. 45, 1723 (1980); Y. Imry, Y. Gefen, D.J. Bergmann, Phys. Rev. B 26, 3436 (1982); A. Kawabata, J. Phys. Soc. Jpn. 53, 318 (1984).

[19] A.M. Finkel'stein, Pis'ma $w$ ZETF 37, 436 (1983); C. Castellani, C. DiCastro, P.A. Lee, M. Ma, S. Sorella, E. Tabet, Phys. Rev. B 33, 6169 (1986).

[20] A.L. Efros, B.I. Shklovski, J. Phys. C 8, L49 (1975).

[21] B. Shapiro, E. Abrahams., Phys. Rev. B 24, 4025 (1981).

[22] H. Kohno, K. Yamada, Prog. Theor. Phys. 80, 623 (1988). 
[23] E. Tousson, Z. Ovadyahu, Phys. Rev. B 38, 12290 (1988); D.W. Koon, T.G. Castner, Phys. Rev. Lett. 60, 1755 (1988); C. Yamanouchi, K. Mizuguchi, W. Sasaki, J. Phys. Soc. Jpn. 22, 859 (1967); R.M. Westervelt, M.J. Burns, P.F. Hopkins, A.J. Rimberg, G.A. Thomas, in Ref. [4], p. 33.

[24] S.B. Field, T.F. Rosenbaum, Phys. Rev. Lett. 55, 522 (1985); M. Rohde, H. Micklitz, Phys. Rev. B 36, 7572 (1987); P. Löbl, M. Esguerra, F. KüB, E. Lüscher, G. Fritsch, M. von Allmen, U. Kambli, A. Schulte, Solid State Commun. 64, 1107 (1987).

[25] J. Wróbel, T. Dietl, G. Karczewski, J. Jaroszyński, W. Plesiewicz, A. Lenard, M. Dybiec, M. Sawicki, Semicond. Sci. Technol. 5, S299 (1990).

[26] T. Dietl, M. Sawicki, T. Wojtowicz, J. Jaroszyński, W. Plesiewicz, L. Świerkowski, J. Kossut, in [4], p. 58.

[27] R.N. Bhatt, M.A. Paalanen, S. Sachdev, J. Physique 49-C8, 1179 (1988) and references therein; M. Milovanović, S. Sachdev, R.N. Bhatt, Phys. Rev. Lett. 63, 82 (1989); M. Lakner, H.v. Lohneyson, Phys. Rev. Lett. 63, 648 (1989); D. Romero, M.-W. Lee, H.D. Drew, M. Shayegan, B.S. Elman, in [4], p. 53. 